\title{
Schizophrenia spectrum and related neuropathology
}

\author{
Andrea Schmitt • Peter Falkai
}

Published online: 28 January 2015

(C) Springer-Verlag Berlin Heidelberg 2015

Focussing on the new DSM-5 classification system, Möller et al. [1] present the second part of their two-piece invited review on changes with reference to the bipolar, schizophrenia spectrum, anxiety and other disorders plus possible consequences under consideration of previous psychiatric classifications. Some important alterations in criteria were made, e.g., in schizophrenia spectrum and related disorders with special consideration of Kurt Schneider's first-rank symptoms. Moreover, the subtypes of schizophrenia have been removed because of insufficient course stability. However, cognitive impairment defined by neuropsychological tests has not been included in DSM-5. Changes in diagnostic criteria are discussed under aspects of rationality, evidence base, usefulness and possible consequences. The new classification system may help to improve differential diagnosis of psychiatric syndromes, but against original intentions, a more neurobiological approach has not been included, probably due to the lack of robust biomarkers for neuropsychiatric disorders. Since schizophrenia is regarded as spectrum disorder, identification of residual symptoms is needed since most patients considered to be remitted by consensus criteria of the remission in Schizophrenia Working Group present mild psychopathological symptoms. In a naturalistic study with 399 schizophrenia patients, Schennach et al. [2] investigated residual symptoms along suggested consensus criteria as defined by any symptom being present at remission and confirmed that indeed, $94 \%$ of the patients suffered from at least one residual symptom,

A. Schmitt $(\varangle) \cdot$ P. Falkai

Department of Psychiatry and Psychotherapy, Ludwig-

Maximilians-University Munich, Nußbaumstr. 7, 80336 Munich,

Germany

e-mail: Andrea.Schmitt@med.uni-muenchen.de mostly blunted affect, conceptual disorganization and social withdrawal. Those symptoms were related to severity of side effects, functioning at discharge and risk of relapse.

Thus, residual symptoms are clinically defined, yet the underlying neuropathology is poorly understood. In a postmortem study of the dorsal raphe nucleus, the main source of serotonergic innervation of forebrain limbic structures, Krzyżanowska et al. [3] found transcriptional activity of ribosomal DNA to be increased in residual versus paranoid schizophrenia patients. This effect was not related to suicide or antipsychotic medication. It may represent a compensatory mechanism to overcome prefrontal serotonergic hypofunction in this patient group. Beside general DNA activity, risk genes such as diacylglycerol kinase eta $(\mathrm{DGKH})$ have been replicated in major psychiatric disorders and gene expression of this variant has been found to be increased in patients with bipolar disorder and schizophrenia. Kittel-Schneider et al. [4] investigated the influence of a risk haplotype of DGKH on the amygdala volume and found an association with increased amygdala volume, a key region implicated in emotional regulation and processing, in bipolar disorder, but not schizophrenia patients or healthy controls. Hass et al. [5] again via fMRI found three complexin2 (CPLX2) SNPs to be associated with increased activity of the dorsolateral prefrontal cortex and intraparietal sulcus in schizophrenia patients compared to controls during a working memory task. This suggests that CPLX2 variants, which are coding for presynaptic proteins involved in neurotransmitter release, may contribute to impaired brain function in schizophrenia. However, to date, it is unclear whether deficits in working memory may also be based on effects of antipsychotic treatment. Potvin et al. [6] investigated symptoms, neurocognition and 
extrapyramidal symptoms in patients with schizophrenia spectrum disorder and performed multivariate hierarchic linear regression analysis. Their results bespeak an association between negative symptoms, antipsychoticinduced parkinsonism and working memory performance. This finding may have clinical implications and strengthens the need for new treatment strategies to specifically improve cognitive deficits. Kerkemeyer et al. [7] describe an integrated care program for schizophrenia in Lower Saxony, Germany, aimed to improve duration of hospitalization, severity of symptoms, remission rates and quality of life.

Cognitive symptoms are based on disturbed connectivity of, e.g., the prefrontal cortex. Using white matter tractography in schizophrenia patients, Iwabuchi et al. [8] showed increased fractional anisotropy in the pathway connecting the right anterior insula with the dorsolateral prefrontal cortex, which was related to reduced effective connectivity. Finally, focussing on the effects of diagnosis and antipsychotic treatment with olanzapine, Ferreira et al. [9] assessed glycogen synthase kinase-3B (GSK-3B) in platelets of drug-free schizophrenia patients at baseline and after 8-week treatment with olanzapine, a group of long-term olanzapine-treated patients and healthy controls. At baseline, drug-free patients showed lower phosphorylated and total GSK-3B levels compared to healthy controls, but after 8 weeks and chronic olanzapine treatment, levels were significantly increased. This enzyme is involved in phosphorylation of proteins and regulation of transcription factors, gene expression, glycogen metabolism and synaptic plasticity, and its dysregulation has been described in postmortem studies in schizophrenia. Since most of the patients in these studies had been treated with antipsychotics, demonstration of effects of olanzapine treatment on GSK-3B is important and relationship with antipsychotic effects should be further investigated.

\section{References}

1. Möller HJ, Bandelow B, Bauer M, Hampel H, Herpertz SC, Soyka M, Maier W (2014) DSM-5 reviewed from different angles: goal attainment, rationality, use of evidence, consequences-part 2 : bipolar disorders, schizophrenia spectrum disorders, anxiety disorders, obsessive-compulsive disorders, trauma-and stressorrelated disorders, personality disorders, substance-related and addictive disorders, neurocognitive disorders. Eur Arch Psychiatry Clin Neurosci. doi:10.1007/s00406-014-0521-9

2. Schennach R, Riedel M, Obermeier M, Spellmann I, Musil R, Jäger M, Möller HJ (2014) What are residual symptoms in schizophrenia spectrum disorder? Clinical description and 1-year persistence within a naturalistic trial. Eur Arch Psychiatry Clin Neurosci. doi:10.1007/s00406-014-0528-2

3. Krzyżanowska M, Steiner J, Brisch R, Mawrin C, Busse S, Braun K, Gos T (2014) Ribosomal DNA transcription in the dorsal raphe nucleus is increased in residual but not in paranoid schizophrenia. Eur Arch Psychiatry Clin Neurosci. doi:10.1007/ s00406-014-0518-4

4. Kittel-Schneider S, Wobrock T, Scherk H, Schneider-Axmann T, Trost S, Zilles D, Reif A (2014) Influence of DGKH variants on amygdala volume in patients with bipolar affective disorder and schizophrenia. Eur Arch Psychiatry Clin Neurosci. doi:10.1007/ s00406-014-0513-9

5. Hass J, Walton E, Kirsten H, Turner J, Wolthusen R, Roessner V, Ehrlich S (2014) Complexin2 modulates working memoryrelated neural activity in patients with schizophrenia. Eur Arch Psychiatry Clin Neurosci. doi:10.1007/s00406-014-0550-4

6. Potvin S, Aubin G, Stip E (2014) Antipsychotic-induced parkinsonism is associated with working memory deficits in schizophrenia-spectrum disorders. Eur Arch Psychiatry Clin Neurosci. doi:10.1007/s00406-014-0511-y

7. Kerkemeyer L, Mostardt S, Biermann J, Wasem J, Neumann A, Walendzik A, Wobrock T (2014) Evaluation of an integrated care program for schizophrenia: concept and study design. Eur Arch Psychiatry Clin Neurosci. doi:10.1007/s00406-014-0508-6

8. Iwabuchi SJ, Liddle PF, Palaniyappan L (2014) Structural connectivity of the salience-executive loop in schizophrenia. Eur Arch Psychiatry Clin Neurosci. doi:10.1007/s00406-014-0547-z

9. Ferreira AS, Raposo NRB, Sallet PC, Van de Bilt MT, MachadoVieira R, Talib LL, Gattaz WF (2014) Lower phosphorylated glycogen synthase kinase-3B levels in platelets of patients with schizophrenia: increment by olanzapine treatment. Eur Arch Psychiatry Clin Neurosci. doi:10.1007/s00406-014-0505-9 